\title{
Quand le propre immeuble de cabinet se transforme en piège fiscal
}

\section{Jean-Pierre Ceccon}

\footnotetext{
* Economie effective grâce au processus d'épargne systématique: - avec un rendement de $3 \%$ Fr. 35 989.- moins l'impôt sur le revenu de $40 \%=$ Fr. 21593 . - avec un rendement de $4 \%$ Fr. 50235.- moins l'impôt sur le revenu de $40 \%=$ Fr. 30141 . - avec un rendement de $5 \%$ Fr. 65 785.- moins l'impôt sur le revenu de $40 \%=$ Fr. 39471 .
}

\section{Correspondance:}

Jean-Pierre Ceccon

Expert diplômé en conseil financier, Planificateur financier CFP

Ceccon Consulting \& Partner AG FMH Treuhand Services

Baselstrasse 10

CH-4222 Zwingen

Tél. 0612610808

Fax 0612610805

jean-pierre.ceccon@fmhtreuhand.ch
Le propre immeuble de cabinet peut s'amortir, tout comme l'inventaire du cabinet. L'amortissement a pour but et finalité de constituer un futur capital d'investissement, p. ex. en vue de l'achat d'un appareil médical. Le législateur exempte cette «épargne en vue d'investissements futurs» de l'AVS et de l'impôt sur le revenu, sous forme $\mathrm{d}^{\prime}$ «amortissements».

Les amortissements concernant le propre immeuble de cabinet doivent être considérés de façon un peu plus différenciée. Ils ne sont en effet pas remplacés après 5 ans, comme c'est p. ex. le cas d'un système informatique. Les premiers frais de rénovation n'apparaissent qu'après 20 à 25 ans. En principe, les amortissements ne devraient pas dépasser les frais de rénovation futurs. Les conséquences des amortissements trop élevés sont décrites ci-après.

Dans cet exemple, il s'agit d'un immeuble habité par le praticien et disposant d'un cabinet au rez-de-chaussée. Le cabinet possède sa propre entrée et ses propres places de stationnement.

La totalité de l'immeuble (partie privée et cabinet) est déclarée comme immeuble commercial. La totalité de la valeur de l'immeuble est donc inscrite à l'actif du bilan du cabinet. Conséquence: l'amortissement est considérablement plus élevé.

La valeur vénale du bien immobilier est aujourd'hui estimée à quelque 3 millions de francs. Au cours des douze dernières années, l'immeuble a été amorti à hauteur d'environ 1 million de francs. La valeur actuelle au bilan est donc de 2 millions de francs.

Grâce à un amortissement d'environ 80000 francs par an, le médecin a pu «économiser» des impôts et des primes AVS d'environ 40000 francs. Il ne faut cependant pas oublier que ces amortissements constituent simplement un report de l'impôt et de l'AVS. Ceux-ci seront dus ultérieurement, au plus tard à la cessation de l'activité lucrative, et ce en une seule fois. L'arrêt de l'activité lucrative se traduit par un transfert du patrimoine commercial au patrimoine privé. Ce transfert entraîne en outre une évaluation de tous les actifs à leur valeur vénale actuelle. La différence entre la valeur comptable et la valeur vénale est assujettie à l'impôt sur le revenu et à l'AVS. Dans cet exemple, l'impôt sur le revenu et l'AVS s'élèvent à environ 500000 francs!

Afin d'économiser en temps utile, à savoir dès le début des amortissements sur la valeur du bien immobilier, l'impôt et la prime AVS différés, l'économie réalisée chaque année devrait être cumulée sur un compte séparé. Avec un horizon de durée supérieur à dix ans, cet argent peut être investi avantageusement sur le marché des placements (obligations/actions). Le rendement ainsi obtenu de 3 à $5 \%$ constitue alors le bénéfice effectif réalisé grâce au report des impôts latents.

L'expérience a cependant montré que l'impôt différé est considéré comme une «économie d'impôts» pure et que cet argent sert principalement à la consommation.*

\section{Conclusion}

- Si vous êtes déjà propriétaire de votre cabinet, nous vous conseillons de faire examiner les conséquences fiscales latentes par un expert chevronné.

- Si vous envisagez l'achat de votre cabinet, nous vous conseillons d'épargner systématiquement les économies annuelles sur un compte/dépôt séparé.

\section{Tableau 1}

Calcul du gain fiscal possible.

\begin{tabular}{lccccc} 
& Année 1 & Année 5 & Année 10 & Année 15 & Total \\
\hline Amortissement & 20000 & 100000 & 200000 & 300000 & 300000 \\
\hline Economie d'impôts et d'AVS & 10000 & 50000 & 100000 & 150000 & 150000 \\
\hline $\begin{array}{l}\text { Empargne avec } \\
\text { - rendement moyen de 3\% }\end{array}$ & 10000 & 53091.36 & 114638.79 & 185989.14 & 185989.14 \\
- rendement moyen de 4\% & 10000 & 54163.23 & 120061.07 & 200235.88 & 200235.88 \\
- rendement moyen de 5\% & 10000 & 55256.31 & 125778.93 & 215785.64 & 215785.64
\end{tabular}

